\title{
A new conservative remap method for discontinuous numerical fields.
}

\author{
Angela Herring ${ }^{1}$, Daniel Shevitz ${ }^{3}$, Eugene Kikinzon ${ }^{3}$, Hoby Rakotoarivelo ${ }^{4, *}$, Jan Velechovsky ${ }^{2}$, \\ Konstantin Lipnikov ${ }^{4}$, Mikhail Shashkov ${ }^{1}$, Navamita Ray ${ }^{3}$ and Rao Garimella ${ }^{4}$. \\ ${ }^{1}$ Continuum models and numerical methods, Los Alamos National Laboratory, USA. \\ ${ }^{2}$ Eulerian codes, Los Alamos National Laboratory, USA. \\ ${ }^{3}$ Applied computer science, Los Alamos National Laboratory, USA. \\ ${ }^{4}$ Applied mathematics and plasma physics, Los Alamos National Laboratory, USA. \\ *corresponding author, email: hoby@lanl.gov
}

\begin{abstract}
Remap aims to accurately interpolate numerical fields from a source to a target mesh. It is an important component in arbitrary Lagrangian-Eulerian simulations in which the mesh evolves between time steps following the fluid displacement, as well as for accurately transferring numerical fields between codes. In this work, we present a new remap method that is conservative, in the sense that the integral of each field on the domain is preserved during the process, while minimizing field perturbations and avoiding diffusion effects on discontinuous fields.
\end{abstract}

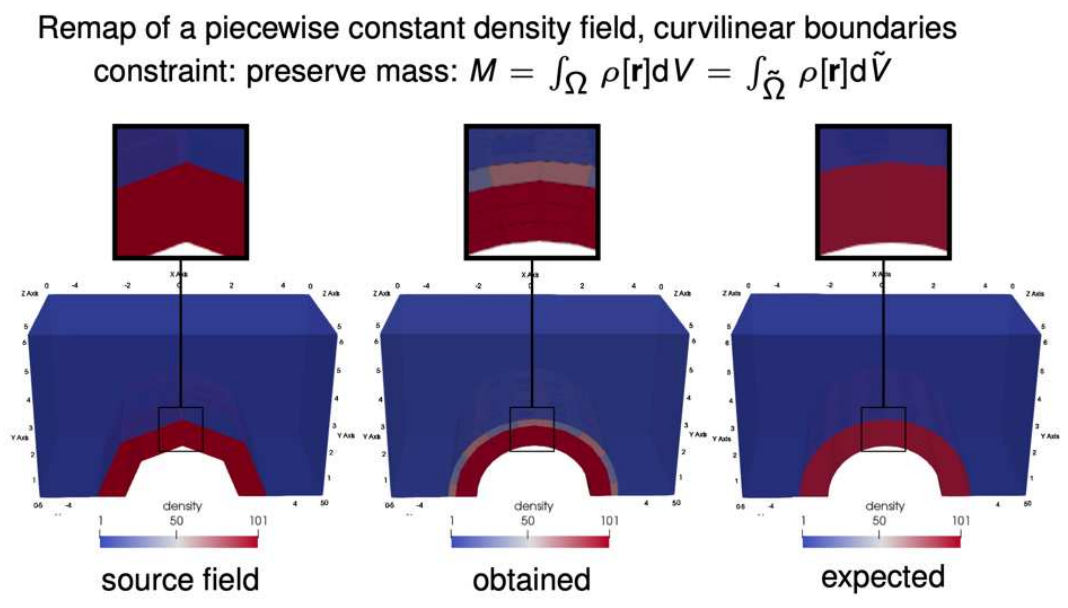

Figure 1 - Diffusion effect induced by the remap of a discontinuous field.

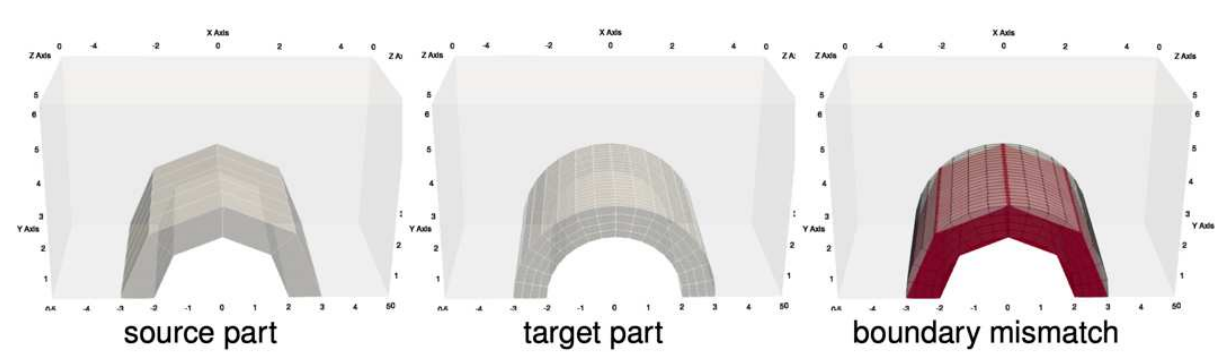

Figure 2 - Decomposition into mesh parts for local remap and mismatch detection.

The problem with discontinuous fields is that serious diffusion effects may occur on the interpolated field as shown in Figure 1. Furthermore, a piecewise constant field may not remain piecewise constant after the remap. To address this issue, the suggested method relies on a local remap between mesh parts, followed by field fixup to enforce conservation and minimize field perturbation. In this context, each mesh part may delineate either a distinct material region or a subdomain delimited by a field value threshold as in Figure 2, and interpolation weights are given by the moments of intersection of a pair of corresponding source and target parts. That said, no assumption is made on those mesh parts which can be topologically arbitrary. In this talk, we first describe the mesh intersection algorithm involved in the computation of the interpolation weights, as well as the boundary mismatch detection. After that, we explain the fixup options used to correct the values on internal and partially overlapped cells, and to extrapolate them on non-overlapped cells. Finally, we present and discuss the numerical results. 